\title{
Idle Retinal Peripheries Diminish Spatial Wrapping
}

\author{
Ahmad Yousef ${ }^{1}$ \\ ${ }^{1}$ School of Computational Science and Engineering, McMaster University, Hamilton, Ontario, Canada \\ *Correspondence: mohamas2@mcmaster.ca
}

\begin{abstract}
This article provides evidence that spatial wrapping can be suppressed by inactive retinal peripheries; a process that can achieved by significant reduction against the contrast between the background and the spatial wrapping stimulus. Significant contrast reduction deactivates the retinal peripheries due to the following reasons; first, the centersurround antagonism in the retinal peripheries may not signal the brain in extremely low contrast conditions. Second, reduced contrast constricts the pupil which disallows the retinal peripheries from receiving sufficient light rays to signal the brain effectively.
\end{abstract}

\section{Introduction}

Spatial wrapping, as shown in the central figure, is a geometrical illusion that curves the straight lines (the dark gray lines) when those lines approach the intersecting node of the background's black lines; an effect that eventually changes the perception of the central flat rectangle to look like a paunch. It has been hypothesized that geometric illusions result from angle overestimation, see reference $1-4$, speculatively as a result of lateral inhibition in visual cortex, see reference 5. Other scientists hypothesized that the wrapping is due to extrapolating $3 \mathrm{D}$ angle information from 2D projections, see reference 6 , and 7. Recent work, however, had suggested that many geometric illusions are caused by temporal delays in the visual cortex, see reference 8, 9 and 10. Creatively, Don Vaughn and David Eagleman had tested the aforementioned hypothesis by implementing random moving dots instead of lines to activate the temporal lobe, astonishingly, human subjects had reported spatial wrapping while the dots are in motion, see reference 11. In this study, however, we may try to suppress the spatial wrapping illusion, through possible deactivations of the retinal peripheries, and thus; we can know whether "the two conscious brains" hypothesis may offer better interpretation to the spatial wrapping phenomenon. It had been reported that low contrast visual stimulation can significantly constrict the pupil, see reference 12; additionally, stimuli that are built with high spatial frequency and low contrast might be able to disallow the retinal ganglion cells in the pripheries to signal the brain (Rosén, etal., 2014). We opportunistically utilized the aforementioned facts trying to deactivate the retinal peripheries. Constricted pupils will disallow a lot of light rays from reaching the retinal peripheries which will reduce the retinal peripheries activations, additionally, the extremely low contrast will greatly vegetate the retinal peripheries' outputs due to the suppressed signal produced by the centersurround antagonism processes. Our preliminary studies show that when the contrast between the stimulus and its background color is extremely low, namely value of 0.1 ; significant suppression against the spatial wrapping illusion could be achieved, see reference 14. Important to mention, many scientists ignore the dynamics between the pupil and lens; dynamics that might greatly affect the spatiotemporal characteristics of the visual awareness. We therefore had critically studied these issues, see reference 15 , and 16; and we tried to link them with the current study. Importantly, the

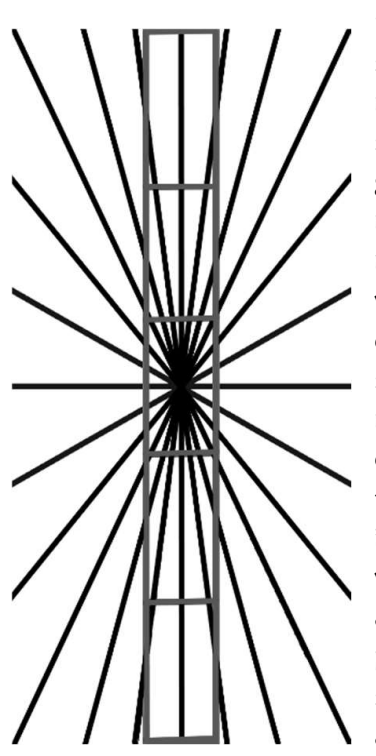
'perceive the present' PTP hypothesis, which stated that temporal delays could be the main reason of the spatial wrapping illusion might be scientifically inaccurate based on our midnight gray stimulus, see reference 14 . This is because the retinal peripheries are assumed to signal the brain much faster than the central retina; and because we observed greater spatial wrapping with high contrast stimuli; stimuli that are considered to significantly dilate the pupil and allows greater retinal peripheries activations but not for low contrast (midnight gray), see reference 14. We therefore will offer another scientific interpretation, namely, we think that spatial wrapping might be diminished if there is no asynchronicity between the central and the peripheral retina, the summation of the output signals from the aforementioned retinal areas is assumed to collectively generate perception of spatial wrapping. Namely, deactivations of the retinal peripheries through pupil constriction plus severely low contrast may cause significant reduction against the temporal asynchronicity between the central and the peripheral retina and its corresponding two integrative conscious brains, see reference 17, 18, and 19; and therefore, possible suppression against spatial wrapping illusion. Namely, we think that spatial wrapping happen, if and only if, there is temporal asynchronicity between the two aforementioned conscious brains; deactivating the peripheral conscious brain, however, might diminish the spatial wrapping. In previous studies, we noted that whether the pupil is naturally constricted "pinpoint pupil", or constricted by the visual stimulus; the 'length dilation' phenomenon, which resembles to spatial wrapping, will be diminished. The magenta and green stimuli that were offered in [19], however, are created to test specific cones, namely, they had similar grayscale contrast, value of 
0.5 ; however, the differences in the perceived dilated length was clear to be noticed. Important to remind, we have choosing human subjects with average size pupils for all of our experiments, and initially excluded the subjects with pinpoint pupils, that is because we had been studying the differences between the central and the peripheral retina and their corresponding conscious brains! We theorized that, because of the anatomical and its stretched zonular fibers, see reference 15; dilated pupils might be the main reason of feeding the fovea but not the retinal peripheries with huge number of light rays that triggers the awareness of length elongation.

\section{Materials \& Methods}

Twelve human subjects were recruited to participate in this study. Each stimulus subtends 8.5 DVA in length, and 4.25 in width. Each subject has to undergo two trials; the first trail presents the central image, in page 1, as a frame of reference; followed by the same stimulus but with a different background color, namely, very dark gray (midnight gray). The aforementioned trail has been flipped for counterbalancing purposes. The midnight gray stimulus now therefore have extremely low contrast. Human subjects has to quantify the reduction of spatial wrapping after seeing the low contrast stimuli compared with the frame of reference. To perfectly quantify all of the aspect of spatial wrapping against the central rectangle we had asked the subjects to estimate the wrapping and the 'pop-up' levels of the aforementioned rectangle; to be compared with the frame of reference. The behavioral data were collected manually, and then averaged among the subjects to estimate the simplest statistical values, namely, the average and the standard error of the mean.

\section{Results and Conclusion}

As shown in the up-central infographic, there is significant degradation against spatial wrapping illusion due to the contrast decrease. Breaking the temporal asynchronicity between the two conscious brains by deactivating the peripheral retina and its corresponding conscious brain seems to diminish the spatial wrapping! We had previously theorized that time and length are coupled, namely, when time dilates, length also has to dilate, see reference 19. In another word, our hypothesis might be literaturely in harmony with the 'perceive the present' PTP hypothesis, namely, temporal delays (time dilation) causes spatial wrapping (length elongation). We, however, now know that the PTP hypothesis had been lacking from certain levels of accuracy; and we think we might had already offered neurophysiological rectifications in the present study. In short, we had studied 'an illusion' though; the present findings may extend into several research points in spatial perception. Essential Notification

Readers should have direct access to the stimuli, see reference 14. Advisably, download the GIFs for the best quality. For rapid validation process, have the test with average pupil, and watch the GIFs in moderate environment; namely, fifty percent of the monitor's max. brightness. Caffeine intake also dilate the pupil, in case of pinpoint pupils. Scholars with brain imaging facilitations are welcome to collaborate in the aforementioned challenging investigations, namely, to localize the spatiotemporal properties of the two conscious brains. Enquiries should be sent to the author.

\section{Transactional References}

[1] Hering, E. (1861). Beitrage zur Physiologie. I. Zur Lehre vom Ortssinne der Netzhaut. Leipzig: Engelmann.

[2] Wundt (1862). Beiträge zur Theorie der Sinneswahrnehmung. Leipzig: Wintersche Verlag.

[3] Holt-Hansen (1961). Hering's illusion. Br. J. Psychol.

[4] Prinzmetal, W., and Beck, D. (2001). The tilt-constancy theory of visual illusions. J. Exp. Psychol. Hum. Percept. Perform.

[5] Blakemore, Carpenter, and Georgeson, (1970). Lateral inhibition between orientation detectors in the human visual system. Nature [6] Nundy, S., Lotto, B., and Coppola, D. (2000). Why are Angles Misperceived? PNAS.

[7] Howe, and Purves (2005). Natural-scene geometry predicts the perception of angles and line orientation. PNAS.

[8] Nijhawan, R. (1997). Visual decomposition of colour through motion extrapolation. Nature.

[9] Changizi, M. A. (2001). 'Perceiving the present' as a framework for ecological explanations of the misperception of projected angle and angular size. Perception.

[10] Changizi, M. A., and Widders, D. M. (2002). Latency correction explains the classical geometrical illusions. Perception.

[11] Don Vaughn and David Eagleman. (2013). Spatial warping by oriented line detectors can counteract neural delays. Front. Psychol.

[12] Wang , and Munoz. (2014) Modulation of stimulus contrast on the human pupil orienting response, in European Journal of Neuroscience. [13] Rosén; etal. (2014). Quick contrast sensitivity measurements in the periphery. In Journal of Vision.

[14] You may find the stimuli in the following link: https://drive.google.com/drive/folders/1S1XPpv68OJJXI7L5SeELFnC492z XZiTn

[15] Yousef, A (2019). "Linking Dynamics Between Pupil and Lens." engrXiv. doi:10.31224/osf.io/9c3vk.

[16] Yousef, A (2019). "The Dynamical Visual Angle." PsyArXiv. doi:10.31234/osf.io/f2kr6.

[17] Sinha, etal. (2017). Cellular and circuit mechanisms shaping the perceptual properties of the primate fovea; in Cell.

[18] Masland (2017) Vision: Two Speeds in the Retina, in Current Biology. [19] Yousef, Ahmad. 2019. "Spatial Attention Dilates Time and Length." PsyArXiv. doi:10.31234/osf.io/qazj9. 